\title{
Lev Vygotski, initiateur du constructivisme social et penseur insaisissable de l'éducation
}

Lev Vygotsky: Initiator of social constructivism and elusive thinker on education Lev Vygotsky, iniciador del constructivismo social y pensador inclasificable del ámbito educativo

Veronika Tašner et Slavko Gaber

Traducteur : Sylvaine Herold

(2) OpenEdition

Journals

Édition électronique

URL : https://journals.openedition.org/ries/7089

DOI : 10.4000/ries.7089

ISSN : 2261-4265

Éditeur

France Education international

Édition imprimée

Date de publication : 1 décembre 2018

Pagination : 109-116

ISBN : 978-2-85420-620-3

ISSN : $1254-4590$

Référence électronique

Veronika Tašner et Slavko Gaber, «Lev Vygotski, initiateur du constructivisme social et penseur insaisissable de l'éducation », Revue internationale d'éducation de Sèvres [En ligne], 79 | décembre 2018, mis en ligne le 01 décembre 2020, consulté le 24 juin 2021. URL : http://journals.openedition.org/ries/ 7089 ; DOI : https://doi.org/10.4000/ries.7089 


\title{
Lev Vygotski, initiateur du constructivisme social et penseur insaisissable de l'éducation*
}

\author{
Veronika Tašner \\ Slavko Gaber \\ Université de Ljubljana
}

\section{UNE POSITION INCLASSABLE \\ PARMI DES DISCIPLINES SCIENTIFIQUES HÉGÉMONIQUES}

Rattacher Vygotski à une seule discipline scientifique relève de l'impossible. L'histoire de son développement professionnel révèle une quête passionnée de compréhension des problèmes qui l'intéressaient. Lev Vygotski naît le 17 novembre 1896 à Orsha, en Biélorussie. Il passe la plus grande partie de son enfance à Gomel, dans une famille de classe moyenne, l'une des familles juives les plus éduquées de la ville. Conformément au vœu de ses parents, Vygotski étudie d'abord la médecine, qu'il abandonne au bout d'un semestre. Au semestre suivant, il s'inscrit en droit à l'Université d'État de Moscou. En parallèle, il suit des cours à l'Université privée Shaniavsky ${ }^{1}$, où il étudie l'histoire et la philosophie. Pendant ses études, il s'intéresse également à la littérature, au théâtre et, surtout, au langage. En 1917, il obtient son diplôme à Moscou et revient à Gomel où il demeure sept ans, enseignant la littérature et l'histoire de l'art.

Avant de s'intéresser à l'apprentissage et la psychologie, Vygotski entre en contact avec le théâtre et la révolution "structuraliste » de Stanislavski. Son cousin David, membre de l'école formaliste de Petrograd, l'initie aux travaux de Roman Jakobson, Lev Jakubinsky et Viktor Shklovsky. En janvier 1924, Vygotski prend part au deuxième congrès panrusse de psycho-neurologie à Léningrad, où il présente trois communications et attire l'attention de Luria et Kornilov. Peu de temps après, ceux-ci l'invitent à devenir chercheur à l'Institut de psychologie de Moscou. Vygotski déménage à Moscou avec sa femme, Roza Smekhova. Fin 1925, il achève sa thèse sur " la psychologie de l'art », ainsi qu'un ouvrage, Psychologie pédagogique. De 1926 à 1930, Vygotski collabore avec Alexander Luria, Boris Varshava, Alexei Leontiev, Leonid Zankov et d'autres encore. Après cette période, il connaît une profonde crise personnelle et théorique, et entreprend une révision radicale de sa théorie. Il décède de la tuberculose le 11 juin 1934 à Moscou.

\footnotetext{
* Article traduit de l'anglais par Sylvaine Herold.

1. A. L. Shaniavsky (1837-1905) est un ingénieur des mines qui s'est enrichi en Sibérie et a utilisé sa fortune afin de financer une université indépendante. Yesenin y a également étudié.
} 
Vygotski a déployé une activité considérable dans un grand nombre de fonctions et d'emplois. Tous étaient liés d'une manière ou d'une autre à l'objectif d'élever le niveau culturel du public en rendant disponible une éducation de haute qualité ainsi que le meilleur de la culture russe et internationale. (Van der Veer, 2007).

Il n'est pas exagéré de dire que ce n'est que dans la dernière partie de sa courte vie, et par pur hasard, que Vygotski trouve finalement sa place dans le domaine de la psychologie, par le biais de l'art². Si la psychologie, en tant que discipline, considère Vygotski avant tout comme un psychologue, il est important de se rappeler, afin de bien comprendre son travail, qu'avant de rencontrer les travaux de Piaget et d'autres grands psychologues de son temps, Vygotski a étudié le droit, la philosophie et l'histoire, et tout particulièrement Descartes, Spinoza, Kant, Hegel, Marx, Husserl, Durkheim, Bruhl, Jakobson, James et Freud. Ces auteurs ont été à l'origine de ses réflexions et de ses travaux pendant sa période « psychologique ».

L'examen du parcours professionnel de Vygotski en tant que chercheur et professeur après la fin de son doctorat, au cours duquel il s'intéresse principalement à la psychologie de l'art ${ }^{3}$, confirme l'impossibilité de le classer au sein d'une seule discipline scientifique ; il considérait d'ailleurs qu'aucune des disciplines scientifiques établies, de par leur caractère exclusif, n'était en mesure répondre à ses attentes en tant que chercheur. Qualifier Vygotski de paidologue pourrait irriter les psychologues, mais le fait est qu'il a conduit des recherches qui dépassaient les divisions conceptuelles établies et qu'il se référait à lui-même en tant que professeur de paidologie. En 1931, il devient professeur de paidologie au Second Institut de médecine de Moscou. C'est à peu près à cette époque que ses travaux subissent leurs premières attaques du fait de liens présumés avec des philosophes et psychologues idéalistes mencheviks. Il continue cependant d'assumer de nouvelles fonctions dans le domaine de la paidologie et, vers la fin de sa vie (janvier 1934), lorsqu'il énumère ses différentes attributions, il n'oublie pas de citer le poste de professeur et directeur du département de paidologie générale et de l'âge au Second institut de médecine de Moscou, ainsi que le poste de professeur et directeur du département de l'enfance anormale à l'Université pédagogique d'État de Moscou (Van der Veer et Valsiner, $1993)$.

Selon Vygotski, le développement de l'enfant ne peut être compris ni analysé en tant que processus de développement biologique, médical, psychologique ou pédagogique particulier. Il est convaincu qu'afin de comprendre le développement de l'enfant, il est nécessaire de relier ces différents facteurs à d'autres, tout en élargissant la perspective afin de comprendre l'enfant non seulement en tant qu'individu mais également en tant qu'être social (un être social et individuel). Vygotski était contemporain de Piaget; il était donc parfaitement au fait des développements de la psychologie de cette époque et il estimait que pour comprendre la formation de l'être humain et le développement de l'enfant, il était nécessaire d'aller au-delà des recherches menées jusqu'alors. Au-delà du processus de développement individuel et biologiquement conditionné de la conscience, Vygotski se lance alors dans des

2. L'art est très important pour Vygotski : « dans sa jeunesse et son enfance, il se tenait au courant des dernières évolutions de la littérature, du théâtre, des beaux-arts et de la musique » (Van der Veer, 2007).

3. Ce travail de doctorat est le résultat de son premier grand projet de recherche, du même titre. Vygotski en remet le manuscrit en 1925. 
recherches sur le développement social de la conscience individuelle. Il est de ce fait considéré de nos jours comme " une figure centrale dans le développement des explications théoriques actuelles de la sociogenèse de la conscience individuelle » (Daniels, 2001).

Mais le projet de Vygotski était en outre de faire de la paidologie une nouvelle discipline scientifique. Van der Veer et Visiner (1993) soulignent qu'un grand nombre de chercheurs ayant étudié Vygotski n'ont pas compris cela. En Union soviétique, cette amnésie a été favorisée par le fait que la paidologie fut dénoncée par décret en 1936 - omettre la paidologie était par conséquent " recommandé » -, mais également car il existait, au sein du monde universitaire, l'ambition de supplanter la paidologie par une combinaison de psychologie et de pédagogie. Ces deux disciplines scientifiques ne renoncèrent évidemment pas à leur position établie dans le champ de la socialisation, même après que Vygotski fut à nouveau « autorisé ».

Ailleurs dans le monde, la paidologie a également été oubliée, sans pour autant avoir été interdite. La réflexion sur la paidologie, telle que Vygotski la concevait, a également été écartée car elle se heurtait à l'interprétation actuelle du constructivisme et, ce qui ne diffère guère de la situation soviétique, du fait du positionnement des disciplines occupant des champs académiques que la paidologie tentait de fusionner en une seule discipline (Bourdieu, 1984).

Il nous paraît intéressant de revenir à cette « discipline interdite et oubliée », non seulement parce qu'elle possède cette largeur de vue que n'ont pas les disciplines qui dominent les études sur la formation des mécanismes, mais également parce qu'elle s'intéresse de près aux problèmes de réussite, d'égalité et de justice en éducation. La paidologie est intéressante également car son développement révèle un type de rationalité typique de l'Union soviétique de l'époque, que Vygotski a adopté (parmi d'autres possibles). Luria (1996) mentionne deux caractéristiques typiques de l'approche de Vygotski : l'holisme et l'historisme. Ce type de rationalité est justement ce qui a permis à la paidologie de dépasser les frontières des disciplines, malgré les restrictions existantes pour le faire, afin d'accéder à une sphère de pensée radicale sur les différents modes d'apprentissage, de pensée et d'être des humains. C'est exactement ce que l'Union soviétique de son temps a accompli dans plusieurs domaines, une décennie et demie après la révolution d'Octobre.

Tout comme Dewey outre-Atlantique, Vygotski a étudié en Union soviétique la question du développement effectif et de la personnalité en accord avec son temps. Ces auteurs aujourd'hui considérés, à juste titre, comme les fondateurs du constructivisme social, ont contribué à la recherche scientifique en éducation à l'époque de l'avènement des sociétés industrielles. Leurs concepts ont permis d'analyser les problèmes des sociétés dans lesquelles l'industrie a fini par dominer l'agriculture en termes de production. La production industrielle transforme les fondements des relations familiales ainsi que les relations entre groupes et classes au sein d'une société. Une nouvelle approche du développement du sujet était dès lors nécessaire, et avec elle le besoin d'une école différente.

4. «Pour Vygotski, l'holisme signifiait la nécessité de rechercher des unités d'activité psychique complexes et structurées, dont la structure est définie par l'ensemble des flux de leur formation au cours du processus de développement ontologique et phylogénétique. L'historicisme signifiait pour Vygotski le récit de l'histoire de la formation des cellules initiales, unités de la psyché » (Luria, 1996). 
En Union soviétique, la période qui suivit la révolution fut marquée par différentes tentatives visant également à mettre en œuvre un changement radical dans l'éducation. Nadezdha Krupskaya fut l'une des principales représentantes du nouvel État socialiste dans le domaine de l'éducation, dans les années vingt. Elle réunit autour d'elle une équipe d'experts, afin de rechercher de nouvelles méthodes d'alphabétisation de la population, dans une large mesure illettrée, et d'inclusion des enfants à besoins spéciaux dans le système éducatif. Leurs principaux objectifs étaient d'accroître l'efficacité du système éducatif, d'élever le niveau de vie de la majorité de la population et de rivaliser avec le capitalisme.

Ces différentes tentatives de réforme furent influencées par les expériences étrangères, mais également par des avancées locales. Ces tentatives pour former « correctement » et " efficacement » les nouvelles générations cherchaient en fait à former un "nouvel homme » et un mouvement en faveur de la nouvelle discipline scientifique dénommée paidologie vit alors le jour. Mais Vygotski, qui connaissait bien la tradition russe en matière d'éducation, souhaitait en faire plus que la simple étude interdisciplinaire du développement de l'enfant. Son ambition était de fonder une discipline scientifique holistique de la socialisation des enfants.

[Il] distingua la paidologie des autres disciplines en la définissant comme la science du développement de l'enfant ». (Van der Veer et Valsiner, 1993)

Dans sa tentative de changer de paradigme, il obtint au moins un succès temporaire : la paidologie fut reconnue pendant un certain temps comme discipline scientifique officielle de la réforme de l'école. À l'apogée du pouvoir de Lunacharsky, alors ministre de l'éducation, et de son adjointe Krupskaya, fut organisé en 1927 le premier congrès soviétique des paidologues, auquel assistèrent plus de trois mille participants, et qui adopta une résolution « appelant à la création d'un nouveau domaine d'études, la paidologie » (Prawat, 2000), ce qui fut célébré par les progressistes en éducation de l'époque. C'est à la même époque que Dewey soutint Lunacharsky, qui était un pragmatique (Prawat, 2000). Mais le succès fut de courte durée.

Les partisans de Staline remportèrent la bataille pour le monopole du développement éducatif en Union soviétique et la paidologie, qui luttait contre une vision étroite du développement de l'enfant en mettant en avant la dépendance sociale des processus psychiques et cognitifs supérieurs, contribuant ainsi à réduire " la complexité psychologique dynamique à ses principaux éléments constituants » (Van der Veer et Valsiner, 1993), en fut la première victime. Ainsi, les auteurs chargés d'éliminer la paidologie du discours public et souhaitant restreindre le domaine du développement de l'enfant à la psychologie et à la psychologie marxiste évitèrent soigneusement le terme paidologie : même après 1956, alors que Vygotski était à nouveau considéré en tant qu'auteur dans le monde universitaire soviétique et qu'il était à nouveau permis de le lire et le publier, le terme de paidologie fut remplacé par «psychologie scolaire », " psychologie de l'enfant » ou simplement «psychologie » (ibid.).

Si l'on s'interroge de nos jours sur les raisons pour lesquelles l'approche de Vygotski était à ce point dérangeante pour que, même après 1956, alors qu'il était à nouveau autorisé, ses propos aient été ainsi " corrigés » en Union soviétique, alors qu'on le considérait comme une grande découverte en Occident, notre thèse est la suivante : la compréhension approfondie que Vygotski proposait de l'influence de la 
cognition sur le langage, en tant que médiateur entre le monde et l'individu, mais également de l'impact de la société sur la cognition, et en particulier de l'influence de ces deux facteurs sur la qualité de la cognition, pouvait paraître à la fois « effrayante et passionnante ». Lorsque Bruner, qui est considéré comme la personne la plus influente ayant diffusé précocement les idées de Vygotski, se remémore sa découverte de cet auteur, il se rappelle notamment une soirée passée avec lui lors d'une rencontre internationale à Montréal, en 1954. Cette rencontre russe, avec "vodka et caviar ", lui révéla que les travaux de Vygotski, qui amélioraient considérablement ceux de Pavlov, étaient « largement diffusés bien qu'officiellement interdits » (Bruner, 1985). Quelques années plus tard, Bruner rédigea la préface de l'ouvrage Pensée et langage de Vygotski. Il raconte la manière dont son étonnement n'avait cessé de croître à la lecture attentive de cet ouvrage : "Vygotski était manifestement génial » (ibid.). Il est révélateur à ce titre que même Bruner ait été d'accord avec la traduction anglaise du titre de l'ouvrage, Thought and Language. C'est encore la traduction qui perdure de nos jours, en anglais, alors qu'il est évident qu'elle est inexacte. Dans l'introduction de 1962, Bruner attire l'attention sur le fait que les travaux de Vygotski ne sont entrés dans le domaine de la psychologie qu'en 1924 (Bruner, 1962). Selon lui, Vygotski est un auteur remarquable car il a été capable d'aller au-delà du comportementalisme, d'une part, et de l'approche subjective des phénomènes cognitifs, d'autre part, qui les considère comme exclusivement subjectifs. Vygotski a de surcroît introduit une perspective historique du développement cognitif et de ce qu'est véritablement la pensée.

Si cet ouvrage ne souleva l'enthousiasme que de quelques psychologues, les sociologues saluèrent indiscutablement les progrès introduits par Vygotski dans la réflexion sur la cognition en termes de mécanismes d'échange entre la société et les fonctions cognitives supérieures de l'individu, et sur les liens existant entre cognition et société.

\section{APPORTS DE VYGOTSKI ET DE SES CONCEPTS EN ÉDUCATION}

Dans sa tentative de s'écarter des explications unidirectionnelles du développement cognitif, encouragé en cela par les aspirations du socialisme révolutionnaire, Vygotski a intégré la société dans sa réflexion sur les outils, le langage et les symboles en tant que facteurs clés de compréhension du développement cognitif chez l'homme. Il montre ainsi qu'au sein du développement cognitif,

deux processus de développement qualitativement différents et d'origine distincte, peuvent être distingués : les processus élémentaires, d'origine biologique, d'une part, et les fonctions psychiques supérieures, d'origine socioculturelle, de l'autre. L'histoire du comportement de l'enfant naît de la rencontre de ces deux processus. (Vygotski, 1978)

Ainsi, les fonctions cognitives supérieures ne se développent pas chez l'enfant sous l'effet de la logique pure. L'enfant ne les invente ni ne les découvre sous la forme de visions ou d'intuitions soudaines suscitant une révélation (l'effet eurêka). 
[...] Au contraire, nous avons constaté que les opérations de signes apparaissent à la suite d'un processus complexe et prolongé, soumis à toutes les lois fondamentales de l'évolution psychologique. Cela signifie que l'activité d'utilisation des signes chez les enfants n'est ni simplement inventée ni simplement transmise par les adultes ; elle découle plutôt de quelque chose qui, à l'origine, n'est pas une opération de signes et ne le devient qu'après une série de transformations qualitatives. (ibid.).

En termes de pédagogie, il est important également que la genèse sociale des fonctions cognitives ne soit pas uniquement centrée sur leurs origines et leur développement, mais que le rôle déterminant de "l'interaction sociale avec l'autre acculturé » soit également pris en compte (Hasan, 1995).

Car, au cours de son processus de développement, l'enfant intériorise les stimuli qu'il reçoit sous forme de discours ou d'outils, ce qui, dans un premier temps, augmente considérablement le «savoir » dont il dispose, ainsi que la vitesse à laquelle celui-ci grandit. Un être humain pense parce que et à condition qu'il transfère les connaissances et compétences accumulées de génération en génération ; il réalise ainsi le potentiel biologiquement transmis. Si l'on reconnaît l'importance de l'accumulation sociale dans la culture, alors la qualité de l'environnement culturel de l'enfant devient également importante pour le développement de sa pensée. À ce titre, différents types d'environnements jouent un rôle de stimulus - avec plus ou moins de succès - pour le développement des fonctions psychiques supérieures de l'enfant ; la réussite scolaire, notamment, est en grande partie liée à ces fonctions. Un autre concept pertinent pour comprendre l'inégalité des résultats scolaires - lié aux fonctions cognitives élémentaires et supérieures, à l'importance du langage (symbole) et de son potentiel d'orientation et de régulation -, également développé par Vygotski et adopté plus tard par les auteurs travaillant dans le domaine de la sociolinguistique et de l'éducation, est le concept de conditionnement précoce, qui se traduit par le développement de la capacité à se soustraire de l'immédiat, du présent, de la réalisation immédiate d'un désir, etc. La principale caractéristique des fonctions élémentaires est d'être déterminée entièrement et directement par des stimuli provenant de l'environnement.

Pour les fonctions supérieures, la caractéristique principale est la stimulation autogénérée, c'est-à-dire la création et l'utilisation de stimuli artificiels qui deviennent les causes immédiates du comportement. (Vygotski, 1978)

La pensée, souligne ainsi Vygotski, devient donc indépendante de l'environnement, ce qui permet à un enfant vivant dans un environnement par ailleurs peu stimulant de négocier différents mondes en dehors de son habitat immédiat. Dans ce cadre, le langage est important en tant que « système symbolique plutôt qu'en tant que dispositif de signes " (Hasan, 1995), comme le souligne un représentant de la sociolinguistique formé selon les vues de Vygotski. Bernstein a également mis en avant l'importance du langage, et en particulier du discours, dans son texte écrit après 1958. Pour lui, le discours - les différents types de discours - et son pouvoir d'orientation et de régulation permet de mesurer le niveau d'intellectualisation de la cognition. À cet égard, l'école représente en soi un outil particulièrement adapté pour accélérer le développement cognitif des enfants, en leur permettant de découvrir de nouveaux mondes, au-delà de l'expérience immédiate. 
Basil Bernstein a découvert Vygotski et Luria en 1958 et a " absorbé la notion de langage en tant que système d'orientation et de régulation » (Bernstein, 1971). Bernstein déclare avoir adopté la recommandation des auteurs en matière de différenciation, qui l'a conduit par la suite, au-delà de la notion de différenciation entre langage public et langage formel, à conceptualiser différents codes.

L'adoption par Bernstein de connaissances issues de l'Union soviétique d'il y a plusieurs décennies est un exemple de réception et d'actualisation sociologiques des concepts de "Vygotski, le paidologue ». Au cours de la dernière décennie de sa vie, alors qu'il était un sociologue accompli, Bernstein est également revenu sur la question de l'importance réelle de Vygotski pour l'évolution de la compréhension du langage, depuis le champ du structuralisme abstrait jusqu'à celui du structuralisme social, contribuant ainsi à développer ses concepts et à étendre l'influence de Vygotski dans le discours contemporain et dans celui de la seconde moitié du xxe siècle.

Comme Bruner, Bernstein a découvert le travail de Vygotski à la fin des années cinquante. Avant la traduction de Pensée et langage, il avait lu une partie de l'ouvrage traduite en anglais par Luria et publiée en 1939 sans grande publicité. Trente-cinq ans plus tard, Bernstein raconte à quel point il fut alors enthousiasmé par sa lecture ${ }^{5}$, affirmant qu'il lui était difficile de décrire l'excitation et le sentiment de révélation que lui procurèrent ce texte ; un nouvel univers s'était ouvert à lui (Bernstein, 1994). Si, à la fin du $\mathrm{xx}^{\mathrm{e}}$ siècle, « le langage et le discours bénéficiaient d'une certaine autorité dans les domaines théorique et appliqué » (ibid.), tel n'était pas le cas à la fin des années cinquante et au début des années soixante. Lorsque, dans son travail Développement linguistique et classe sociale: une théorie sociologique de l'apprentissage, il posait les questions suivantes : 1) «de quelle façon une structure sociale particulière devient-elle partie intégrante de l'expérience individuelle ?»;2) «par quel processus cela advient-il ? » et 3 ) «quelles sont les implications de ce processus pour l'éducation? », Bernstein (1961) entrait dans une zone inconnue. Il s'appuyait certes déjà sur Vygotski et sur Luria ${ }^{6}$, mais il posait également une question qui, comme Vygotski à son époque, paraissait étrange et à peine plausible. Il faut se rappeler que l'époque était alors au développement accéléré des établissements d'enseignement secondaire et aux investissements massifs dans l'éducation à travers toute l'Europe. Le nationalisme économique présentait l'école comme la solution à plus ou moins tous les problèmes du monde d'alors. Il est vrai que l'école devrait éliminer peu ou prou toutes les inégalités sociales. Mais on réalise à quel point les idées de Bernstein sur le langage et sur l'école, inspirées de Vygotski, étaient audacieuses et originales, lorsqu'on se souvient du silence de la gauche et de la droite, de la classe ouvrière et des propriétaires d'usines, des dirigeants et présidents de syndicats, suite à la publication de l'ouvrage de Bourdieu et Passeron en 1964, Les héritiers, dans lequel ces auteurs pointent du doigt les attentes irréalistes vis-à-vis de l'école en tant que mécanisme principal d'élimination des inégalités sociales. En affirmant que l'école n'éliminait évidemment pas les inégalités, ces auteurs furent jugés suspects et devinrent la cible des partisans du gaullisme comme des communistes.

5. Cet extrait (qui semble être le chapitre 7) a été publié en 1939 sous le titre Pensée et discours, une traduction conforme à l'original, contrairement à la traduction inexacte ultérieure du titre du livre.

6. Une comparaison du texte publié en 1961 avec celui intitulé Quelques déterminants sociologiques de la perception (Bernstein 1971/2009), publié pour la première fois en 1958 et qui a servi de base au texte de 1961, révèle qu’une partie a été intégrée au texte révisé, puisque Vygotski et Luria figurent parmi les références. 
Il n'est donc guère surprenant que, trente ans plus tôt, en 1934, dans une Russie alors dirigée par Staline, Vygotski ait été à ce point incompris et en avance sur son temps. Ses idées devraient de nos jours faire partie intégrante des efforts en faveur d'une éducation plus équitable et plus efficace pour tous, notamment ceux issus des classes socialement défavorisées et d'environnements linguistiques différents. Une école qui prendrait en compte son avertissement sur l'importance de l'environnement linguistique - l'importance des formes idéales communiquées aux enfants au cours de leur processus de formation - incarnerait un mécanisme de développement cognitif conforme à son concept de zones proximales de développement. Cela pourrait constituer l'héritage contemporain du grand paidologue, mais également une nouvelle forme de responsabilité et de soutien pour les générations actuelles d'enseignants en faveur d'une école plus juste pour tous.

\section{BibLIOGRAPHIE}

BOURDIEU P. (1984) : Homo academicus, Paris : Les éditions de minuit.

DANIELS H. (2001) : "Bernstein and Activity Theory », dans Towards a Sociology of Pedagogy, New York, Peter Lang.

BERNSTEIN B. (1958/1961) : « Social class and linguistic development: a theory of social learning ", dans A. H. Halsey, J. Floud dans C. A. Anderson (ed.), Education, economy, and society (p. 288-314), New York, The Free Press.

BERNSTEIN B. (1971) : Class, Codes and Control, Theoretical Studies towards a Sociology of Language (Livre I), Londres, Routledge.

BERNSTEIN B. (1994) : "Foreword ", dans H. Daniels (coord.), Charting the agenda. Educational activity after Vygotsky, New York, Routledge.

BRUNER J. (1985) : "Vygotsky: a historical and conceptual perspective ", dans J. V. Wertsch (coord.), Culture, communication, and cognition: Vygotskyan perspectives, Cambridge, CUP.

HASAN R. (1995) : «On Social Conditions for Semiotic Mediation: The Genesis of Mind in Society ", dans A. R. Sadovnik (coord.), Knowledge and Pedagogy: The Sociology of Basil Bernstein, Londres, Abley Publishing.

LURIA A. R. (1996) : " Pogovor ", dans L. S. Vygotsky, Sabrana dela tom drugi: Problemi opšte psihologije, Beograd, ZUNS.

PRAWAT R. S. (2000) : " Dewey Meets the "Mozart of Psychology" in Moscow: The Untold Story ", American Educational Research Journal, vol. 37, n 3, p. 663-696.

VAN DER VEER R. (1993) : "Understanding Vygotsky: A Quest for Synthesis », dans J. Valsiner, Oxford, Blackwell.

VYGOTSKY L. S. (1978) : Mind in Society. The Development of Higher Psychological Processes, édité par M. Cole et al., Londres, HUP.

VYGOTSKY L. S. (1986) : Thought and Language, Massachusetts, MIT. 